\title{
Early postpartum lactation effects of cesarean and vaginal birth
}

\author{
Yuksel İsik, Zeynep O. Dag, Ozlem B. Tulmac, Eren Pek \\ Department of Obstetrics and Gynecology, Faculty of Medicine, Kirikkale University, Kirikkale, Turkey
}

\begin{abstract}
Objectives: Breastfeeding has positive effects for both, the mother and the infant. The purpose of the study was to examine how cesarean delivery and vaginal delivery influenced subsequent breastfeeding. The study was conducted at the Kırıkkale University Medical School.

Material and methods: Breastfeeding outcomes after an elective cesarean delivery and after a planned vaginal delivery were compared. The study included 169 consenting mothers who gave birth to healthy infants ( 86 cesarean deliveries and 83 vaginal deliveries) between March and September 2001. All cesarean deliveries were performed under regional anesthesia.

Results: Elective cesarean delivery was performed at a significantly earlier gestational age as compared to vaginal delivery $(p=0.001)$. Maternal age in the planned vaginal delivery group was significantly lower $(p=0.003)$. As for the change in prolactin levels, the results were similar but not statistically significant $(p=0.21)$. The frequency of breastfeeding per day did not differ significantly between the groups $(p=0.20)$. However, women after cesarean delivery tended to breastfeed more often than after vaginal delivery $(p=0.003)$. Mean number of points recorded at the first breastfeeding session, according to the LATCH charting system, was lower in the group after cesarean delivery as compared to vaginal labor. The difference between the average point scores of vaginal delivery and cesarean delivery mothers was found to be meaningful in favor of the women after vaginal delivery $(p=0.05)$.
\end{abstract}

Conclusions: Elective cesarean section has negative effects on breastfeeding. Our results indicate that cesarean section constitutes a risk factor for delayed lactogenesis.

Key words: breastfeeding, elective cesarean delivery, planned vaginal delivery, prolactin

Ginekologia Polska 2016; 87, 6: 426-430

\section{INTRODUCTION}

Human milk, which has proven health benefits, is considered to be the most suitable food source for all infants. The World Health Organization (WHO), the American College of Obstetricians and Gynecologists (ACOG), the American Academy of Pediatrics (AAP), and the United States Preventive Services Task Force all recommend breastfeeding for the first 6 months of neonatal life [1-4]. Hospital policies play a major role in the success of breastfeeding. One study reported that $30 \%$ of mothers whose intention was to breastfeed exclusively were not successful in reaching this goal [5]. Lactogenesis is a two-stage process: stage I (secretory initiation) - during the second half of pregnancy, and stage II (secretory activation) — after delivery. Stage II lactogenesis occurs between day
2 and 4 postpartum, and is marked by the swelling of the mother's breasts and the onset of copious milk production. If failure to lactate occurs during that stage, breastfeeding can be delayed up to 7-10 days postpartum [6]. Immediately after birth, the infant should be given to the mother for skin-to-skin contact, unless there are medical problems [4]. During the postpartum period, physical contact between the mother and the infant, as well as contact between the infant's lips and the nipple, are associated with increased initiation and duration of breastfeeding, and the latter should be started in the first hour after delivery. If necessary, professional help should be given to the mother during breastfeeding. Parents should be provided with postnatal educational and support programs which promote breastfeeding before and after birth, during hos- 
pitalization, and after discharge. The nursing mother must be in a comfortable position while breastfeeding. Latch-on is facilitated by supporting the breast in one hand with four fingers underneath, and the thumb on top of the breast. Efficient transfer of milk requires coordination of suckling and swallowing. During breastfeeding, ankyloglossia, also known as 'tongue-tie', occurs when the frenulum connecting the tongue to the floor of the mouth is tight and limits extension of the tongue. It is a relatively common finding in newborns and the majority of infants with tongue-tie are capable of breastfeeding without difficulty. Infants who have problems with breastfeeding after lactation is established should be examined for ankyloglossia [7]. If the delay in lactogenesis lasts longer than 4 days, mother-infant dyads require careful monitoring and extra support [8]. There is evidence to suggest that the initiation rates of breastfeeding are lower in infants born by cesarean section (CS), as compared to vaginal delivery (VD). However, there is no difference in breastfeeding rates between infants born by VD and those delivered via CS at 6 months of age. General anesthesia used for CS may decrease alertness of the infant and interfere with initiation of nursing. However, this is an infrequent problem as most cesarean deliveries are performed under local anesthesia. Infants born by VD or CS can be given to their mothers for skin-to-skin contact in the operating room $[9,10]$.

\section{MATERIAL AND METHODS}

This observational and comparative study was conducted between March 4, 2014 and April 26, 2015 at the Obstetrics and Gynecology (Ob-Gyn) Department of a hospital affiliated with the Kırıkkale University Medical School, Kırıkkale, Turkey. Breastfeeding patterns of 169 consenting mothers, who gave birth to healthy infants between March-September 2001, were assessed by comparing cesarean $(n=86)$ and vaginal $(n=83)$ deliveries.

First, the differences in characteristics, lactation outcomes, and prolactin concentrations between primiparas who had a VD and either primiparas who had a cesarean section (CS) or multiparas who had a VD (none of the multiparas had a CS), were examined. The differences between independent variables and lactation outcomes were assessed. All CS deliveries were performed under local anesthesia, and all infants delivered by CS were given to their mothers for skin-to-skin contact in the operating room. Repeat caesareans were included, but emergency cesareans were excluded. Indications for elective CS included repeat CS and other causes of uterine scarring (i.e., myomectomy), malpresentation, macrosomia, and dystocia. VD was defined as regular uterine contractions with progression of cervical dilatation. Local Ethics Committee approved of the study and all participants gave their written informed consent.
The hospital in which the study was conducted was established in 1998 and has a 300-bed capacity. There are 40 private rooms in the $\mathrm{Ob}-\mathrm{Gyn}$ department, which included 15 nurses, and is located on the same floor as the neonatal section. The neonatal section comprises 20 beds and 14 intensive care units, and is staffed by a neonatologist, three specialist physicians, and 12 nurses.

Eligibility criteria for participation involved the following factors: maternal age of 18-35, residents of Kırıkkale for $>1$ year, with Turkish literacy skills and no history of serious medical conditions or obstetrical complications. Infant eligibility criteria included: gestational age of between $37(0 / 7)$ and $41(0 / 7)$ weeks, as determined by the physician and as documented in the medical records, an Apgar score of at least 8 at 5 min., and the condition of being cared for in a normal nursery with no complicating factors. None of the participating mothers smoked cigarettes during the third trimester or during the first day postpartum, or had diabetes. All patients had normal results in glucose tolerance tests during pregnancy.

Blood samples were obtained from mothers undergoing CS and planned VD on day 1 postpartum, in order to measure prolactin concentrations. On the day after delivery, before the second breastfeeding between 09:00 $\mathrm{h}$ and 11:00 h, an intravenous cannula was inserted into the antecubital vein. Each blood sample took approximately 15-20 s to draw, and samples were collected by venipuncture after an overnight fast. Some of the blood (for plasma samples) was transferred into a tube with ethylene glycol-bis-N, N9-tetraacetic acid.

Characteristics of breastfeeding were assessed according to the LATCH charting system [11], with five sub-groups adapted for Turkey by Yenal and Okumus [12], on the basis of the original system developed by Jensen et al. [11]. The assessment of the breastfeeding session is made on the basis of the total score. A total score of $<10$ indicates that the mother is in need of support [12]. The letters of the acronym LATCH designate separate areas of assessment: $L$ for how well the infant latches onto the breast, A for the amount of audible swallowing, $T$ for the mother's nipple type, $C$ for the mother's level of comfort, and $\mathrm{H}$ for the amount of support the mother has to be given in order to hold her infant to the breast. The LATCH findings for the first two breastfeeding sessions of each mother recorded on the breastfeeding monitoring card are presented in Table 1.

\section{Statistical analysis}

All clinical parameters were expressed as mean $\pm S D$, as well as percentages (for categorical variables). The participants were compared using a repeated measures analysis of variance for continuous parameters and a Chi-square test for categorical parameters. The $p$-value of $<0.05$ was consid- 


\begin{tabular}{|c|c|c|c|}
\hline \multirow{2}{*}{ LATCH } & \multicolumn{3}{|c|}{ SCORE } \\
\hline & 0 & 1 & 2 \\
\hline $\begin{array}{l}\text { L } \\
\text { LATCH }\end{array}$ & $\begin{array}{l}\text { Too sleepy or reluctant } \\
\text { No sustained latch or suck achieved }\end{array}$ & $\begin{array}{l}\text { Repeated attempts for sustained } \\
\text { latch and suck } \\
\text { Hold nipple in mouth } \\
\text { Stimulate to suck }\end{array}$ & $\begin{array}{l}\text { Grasps breast } \\
\text { Tongue down } \\
\text { Lips flanges } \\
\text { Rhytmical sucking }\end{array}$ \\
\hline $\begin{array}{l}\text { A } \\
\text { AUDIBLE SWALLOWING }\end{array}$ & None & A few with stimulation & $\begin{array}{l}\text { Spontaneous and intermittent }<24^{\circ} \\
\text { Spontaneous and frequent }\end{array}$ \\
\hline $\begin{array}{l}\text { T } \\
\text { TYPE OF NIPPLE }\end{array}$ & Inverted & Flat & Everted (after stimulation) \\
\hline $\begin{array}{l}\text { C } \\
\text { COMFORT (Breast-Nipple) }\end{array}$ & $\begin{array}{l}\text { Engorged } \\
\text { Cracket, bleeding, Ig. blisters or } \\
\text { bruises } \\
\text { Severe discomfort }\end{array}$ & $\begin{array}{l}\text { Filling } \\
\text { Reddened/small blisters or bruises } \\
\text { Mild-moderate discomfort }\end{array}$ & $\begin{array}{l}\text { Soft } \\
\text { Non-Tender }\end{array}$ \\
\hline $\begin{array}{l}\text { H } \\
\text { HOLD (Positioning) }\end{array}$ & Full assist (staff holds) & $\begin{array}{l}\text { Minimal assist (pillows, } \uparrow \text { HOB) } \\
\text { Teach one side, Mom does other } \\
\text { Staff holds } \rightarrow \text { Mom takes over }\end{array}$ & $\begin{array}{l}\text { No assist from staff } \\
\text { Mom able to position/hold baby }\end{array}$ \\
\hline
\end{tabular}

\begin{tabular}{|l|l|l|}
\hline \multicolumn{2}{|l|}{ Table 2. Charecteristics of women with elective cesarean section and with planned vaginal delivery } \\
\hline Characteristic of subjects & Planned VD (n= 83) (\%) & Elective CS (n= 86) (\%) \\
\hline Maternal age $(p=0.003)$ & $25.14 \pm 5.68$ & $27.19 \pm 5.41$ \\
\hline Gestational week $(p=0.001)$ & $39.24 \pm 1.16$ & $38.43 \pm 0.97$ \\
\hline Parity & & $33(38.37)$ \\
\hline \multicolumn{1}{|c|}{ Primiparous } & $34(40.96)$ & $53(61.02)$ \\
\hline \multicolumn{1}{|c|}{ Multiparous } & $49(59.03)$ & $45(52.32)$ \\
\hline Sex of infants (\% males) & $45(54.21)$ & \\
\hline VD - vaginal delivery:CS - & &
\end{tabular}

ered as statistically significant. Data analysis was conducted using the SPSS (version 11.0) statistical package.

\section{RESULTS}

The mothers were divided into four groups, as follows: primiparous women undergoing CS, multiparous women undergoing $C S$, primiparous women undergoing VD, and multiparous women undergoing VD. All CS deliveries were performed under local anesthesia.

Deliveries were classified as VD and elective CS. Patient characteristics (maternal age, gestational week, parity, and gender of the infants) were comparable between the two groups and are presented in Table 2. Neither vacuum extractor nor forceps were required in the planned VD group, and no major intraoperative trauma was recorded in the elective CS group. Elective CS was performed at a significantly earlier gestational age than VD $(p<0.001)$, and maternal age was significantly lower for planned VD $(p<0.003)$. Data relating to the planned VD group are presented in Table 2 , and planned VD was achieved in $40.96 \%$ of the primiparous, and $59.03 \%$ of the multiparous mothers. The rate of male infants was $54.21 \%$. In the elective CS group, the proce- dure was performed under spinal anesthesia, and $38.37 \%$ had primiparous pregnancy, while $61.02 \%$ had multiparous pregnancy. The rate of male infants was $52.32 \%$, as shown in Table 2.

The characteristics of breastfeeding according to the LATCH charting system are shown in Table 1. A comparison of mean points recorded with regard to planned VD and elective CS at the first breastfeeding session revealed that there was a significant difference in favor of the mothers undergoing planned VD.

Infant birth weight did not significantly differ between the four groups $(p=0.15)$. There were no significant differences in the Apgar score at 1 and $5 \mathrm{~min}$. $(p=0.48$ and $p=0.61$, respectively). The time of the first feeding was similar in the four groups, and was not statistically significant ( $p=0.34$ ). Babies born by CS were less likely to be feeding at their first maternal contact after birth, and their mothers were less likely to hold them directly against their own naked skin (first feeding time was a mean of 83.82 minutes). These mothers were more likely to require help to initiate breastfeeding. Breast selection was similar in the four groups, and was generally the left breast. The frequency of feeding on 


\begin{tabular}{|c|c|c|c|c|c|}
\hline Independent variables & Primiparous CS & Multiparous CS & Primiparous VD & Multiparous VD & p \\
\hline Infant birth weight [g] & $3402,12 \pm 592.84$ & $3222.26 \pm 468.05$ & $3210.29 \pm 350.19$ & $3266.71 \pm 622.04$ & 0.15 \\
\hline \multicolumn{6}{|l|}{ Apgar score } \\
\hline $1 \mathrm{~min}$ & $8.58 \pm 0.66$ & $8.41 \pm 0.57$ & $8.58 \pm 0.49$ & $8.58 \pm 0.53$ & 0.48 \\
\hline $5 \mathrm{~min}$ & $9.46 \pm 0.63$ & $9.26 \pm 0.61$ & $9.44 \pm 0.61$ & $9.56 \pm 0.60$ & 0.61 \\
\hline Tie of first feeding (min postpartum) & $87.27 \pm 33.84$ & $80.37 \pm 31.00$ & $30.00 \pm 0.01$ & $30.00 \pm 0.01$ & 0.34 \\
\hline Breastfeeding frequency, day 1 [h] & $9.81 \pm 1.57$ & $8.98 \pm 1.08$ & $8.67 \pm 0,87$ & $9.06 \pm 0.96$ & 0.20 \\
\hline Breast selection (frequency [\%]) & $\begin{array}{l}\text { left breast } \\
(71.42 \%)\end{array}$ & $\begin{array}{l}\text { left breast } \\
(65.21 \%)\end{array}$ & $\begin{array}{l}\text { left breast } \\
(82.35 \%)\end{array}$ & $\begin{array}{l}\text { left breast } \\
(81.13 \%)\end{array}$ & \\
\hline $\begin{array}{l}\text { Serum prolactin }[\mathrm{ng} / \mathrm{ml}] \text {, day } 1 \\
\text { postpartum }\end{array}$ & $326.21 \pm 109.66$ & $284.84 \pm 119.22$ & $294.50 \pm 89.55$ & $295.95 \pm 106.81$ & 0.21 \\
\hline \multirow[t]{2}{*}{ Breastfeeding charting system (LATCH) } & $7.18 \pm 1.55$ & $6.98 \pm 1.35$ & $7.41 \pm 1.39$ & $7.61 \pm 1.44$ & 0.37 \\
\hline & \multicolumn{2}{|c|}{ Cesarean } & \multicolumn{2}{|c|}{ Vaginal Delivery } & \\
\hline Time of first feeding (min postpartum) & \multicolumn{2}{|c|}{$83.82 \pm 32.10$} & \multicolumn{2}{|c|}{$30.00 \pm 0.01$} & 0.001 \\
\hline Breastfeeding frequency, day 1 [h] & \multicolumn{2}{|c|}{$9.40 \pm 1.34$} & \multicolumn{2}{|c|}{$8.86 \pm 0.94$} & 0.003 \\
\hline Breastfeeding charting system (LATCH) & \multicolumn{2}{|c|}{$7.08 \pm 1.42$} & \multicolumn{2}{|c|}{$7.51 \pm 1.41$} & 0.05 \\
\hline
\end{tabular}

All values are displayed as means or frequency [\%]; $x \pm S D$; significantly different from pregnancy, within category, $p \leq 0.05 ; C S-$ elective cesarean; VD - planned vaginal delivery

the first day was not significantly different between the four groups $(p=0.20)$. However, a tendency was observed for babies delivered by CS to be fed more often than those born by VD ( $p=0.003)$. The change in prolactin levels between the four groups was similar but not statistically significant $(p=0.21)$, and the overall rates were high (Table 3 ).

Mean number of points recorded at the first breastfeeding session was lower after CS as compared to VD, according to the LATCH charting system. There were no significant differences $(p=0.37)$ in the rate of mean points between infants born by primiparous vaginal, multiparous vaginal, primiparous caesarean, and multiparous cesarean delivery (Table 3). The difference between the point averages of mothers undergoing VD and those who delivered via CS was meaningful in favor of the mothers who gave birth vaginally $(p=0.05)$.

\section{DISCUSSION}

In our study, detailed records of early feeding behavior were obtained. In addition, biochemical markers of lactogenesis were assessed. CS was shown to affect breastfeeding as CS mothers were more likely to require support in this respect as compared to mothers after VD. Elective CS (even under spinal anesthesia) was demonstrated to be negatively associated with breastfeeding.

Breastfeeding of mothers undergoing elective CS was assessed and the outcome was compared with breastfeeding of mothers who had a planned VD in low-lactation. A significantly greater number of breastfeeding problems during the postpartum period was observed in the elective CS group. Primiparous and multiparous women after
CS tended to breastfeed less often after the first day postpartum than primiparous and multiparous women after VD. However, the analysis of potentially confounding variables revealed that parity was not a significant risk factor for breastfeeding. CS delivery and the period after birth are other potential determinants of the timing of lactogenesis, but their effects are also difficult to disentangle. The results of our study indicate that elective CS (under spinal anesthesia) and planned VD can have a similar effect on breastfeeding during the early postpartum period. Our study also showed that mothers in general need more professional support as far as breastfeeding is concerned, and that this need is more pronounced in mothers after a CS birth.

Optimal delivery room care includes skin-to-skin contact between the mother and the infant, breastfeeding within the first hour after birth, and delay of any unnecessary interventions until after this initial period of mother-infant interaction. Some researchers have claimed that early postpartum discharge from hospital is beneficial for the mother-infant relationship and subsequent breastfeeding [13]. Physical contact between the mother and the infant in the immediate postpartum period, and contact between the infant's lips and the nipple are associated with increased initiation and duration of breastfeeding [4]. There is evidence that the initiation rates of breastfeeding are lower in CS infants as compared to those born vaginally. In a meta-analysis, the rate of initiation of breastfeeding was lower after CS versus VD [14].

In one systemic review, a subgroup analysis suggested that the decrease in early breastfeeding occurred primarily due to delivery by elective CS, as the rates of breastfeeding initiation in infants born by emergency CS and those 
delivered vaginally were comparable [4]. Most CS deliveries are performed under local anesthesia, and the majority of infants delivered in this manner can be given to their mothers for skin-to-skin contact already in the operating room. Mothers who undergo CS may need additional help from the clinical staff to attain comfortable and correct positioning of their infant for breastfeeding [15].

Breastfeeding has direct clinical benefits for the infants, as well as potential-long term benefits after the breastfeeding period is over, including improvement in the host defense and gastrointestinal function, and prevention of acute illnesses. Long-term benefits are improved intelligence-test scores [16], as well as a lower risk of subsequent specific chronic diseases and hospitalization. The published data strongly support breastfeeding for all infants [17].

In summary, our findings indicate that CS constitutes a risk factor for delayed lactogenesis. The results from the early postpartum period showed that women who have CS experience sub-optimal birthing and early parenting outcomes. Our findings are consistent with those previously reported. However, similar studies carried out in different regions of the world and with ethnic groups of mothers are warranted.

\section{REFERENCES}

1. World Health Organization. Global Strategy for Infant and Young Child Feeding 2003.www.who.int/nutrition/publications/infantfeeding/en/index.html. Accessed on April 10, 2009.

2. PubMed. Primary care interventions to promote breastfeeding: U.S. Preventive Services Task Force recommendation statement. U.S. Preventive Services Task Force. Ann Intern Med. 2008, 560, 149-158.
3. ACOG Committee Opinion No. 361: Breastfeeding: maternal and infant aspects. Committee on Health Care for Underserved Women, American College of Obstetricians and Gynecologists. Obstet Gynecol. 2007, 479, 109-112.

4. Breastfeeding and the use of human milk. Section on Breastfeeding Pediatrics 2012, 827, 129-133.

5. Perrine CG, Scanlon KS, Li R, Odom E, Grummer-Strawn LM. Baby-Friendly hospital practices and meeting exclusive breastfeeding intention. Pediatrics. 2012, 54, 130-131.

6. Brownell E, Howard CR, Lawrence RA, Dozier AM. Delayed onset lactogenesis II predicts the cessation of any or exclusive breastfeeding. J Pediatr. 2012, 608, 161-164

7. American Academy of Pediatrics Committee on Nutrition. Breastfeeding In: Nutrition Handbook, 6th ed. Kleinman RE (ed), American Academy of Pediatrics, Elk Grove 2009, 29.

8. Shao Y, Zhao FQ. Emerging evidence of the physiological role of hypoxia in mammary development and lactation. J Anim Sci Biotechnol. 2014, 21, 1-9.

9. Prior E, Santhakumaran S, Gale C, Philipps LH, Modi N, Hyde MJ. Breastfeeding after cesarean delivery: a systematic review and meta-analysis of world literature. Am J Clin Nutr. 2012, 95, 1113-1135.

10. McDonald SD, Pullenayegum E, Chapman B, [et al.]. Prevalence and predictors of exclusive breastfeeding at hospital discharge. Obstet Gynecol. 2012, 119, 1171-1179.

11. Jensen D, Wallace S, Kelsay P. LATCH: Breastfeeding Charting System and Documentation Tool. J Obstet Gynecol Neonatal Nurs. 1994, 23, 27-32.

12. Yenal K, Okumus E. Reliability of LATCH breastfeeding assesment tool. Turkish Journal of Research and Development in Nursing. 2003, 1, 38

13. Wright CM, Parkinson $\mathrm{K}$, Scott J. Breast-feeding in a UK urban context: who breast-feeds, for how long and does it matter? Public Health Nutr. 2006, 9, 686-691.

14. Prior E, Santhakumaran S, Gale C, Philipps LH, Modi N, Hyde MJ. Breastfeeding after cesarean delivery: a systematic review and meta-analysis of world literature. Am J Clin Nutr. 2012, 95, 1113-1135.

15. Zanardo V, Pigozzo A, Wainer, [et al.]. Early lactation failure and formula adoption after elective caesarean delivery: cohort study. Arch Dis Child Fetal Neonatal Ed. 2013, 98, 37-41.

16. Belamarich PF, Bochner RE, Racine AD. A Critical Review of the Marketing Claims of Infant Formula Products in the United States. Clin Pediatr (Phila). 2015, pii: 0009922815589913

17. Ajetunmobi OM, Whyte $B$, Chalmers J, [et al.]. Breastfeeding is associated with reduced childhood hospitalization: evidence from a Scottish Birth Cohort (1997-2009). J Pediatr 2015, 166, 20. 